\title{
Safety and tolerability of duloxetine - adverse events and how to handle them
}

\author{
Peter Haddad \\ From International Society on Brain and Behaviour: 2nd International Congress on Brain and Behaviour \\ Thessaloniki, Greece. 17-20 November 2005
}

The efficacy and side effect profile of antidepressants are important issues to consider when selecting an antidepressant for a patient. Duloxetine is a serotonin (5HT) and noradrenalin (NE) reuptake inhibitor (SNRI) that has recently been licensed for the treatment of depression. This presentation concerns duloxetine's side effect profile.

Before considering side effects seen in clinical studies it is helpful to consider the pharmacokinetic and pharmacodynamic properties of duloxetine. Duloxetine is 96\% protein bound and has an elimination half-life of about 12 hours. Food has no clinically significant effect on its absorption. Duloxetine is metabolized by CYP2D6 and CYP1A2. Duloxetine does not inhibit CYP1A2 or CYP3A4 but it is a moderate inhibitor of cytochrome CYP2D6. According to published data, duloxetine is a less potent inhibitor of CYP2D6 than paroxetine, but a more potent inhibitor of CYP2D6 than sertraline. Duloxetine has no major active metabolites.

Studies in rats show that following a dose of duloxetine, there are dose dependent increases in both 5HT and NE in the frontal cortex as measured by microdialysis. Even at lower doses there were measurable increases in both neurotransmitters. The similarity of the $5 \mathrm{HT}$ and NE curves suggests that effects on both neurotransmitters were present at all doses and rose according to the dose given.

In clinical trials most side effects seen with duloxetine are mild and transitory. Side effects that occur in duloxetine-treated patients at a rate of $>5 \%$ and twice the rate for placebo are (in descending order of incidence) nausea, dry mouth, constipation, fatigue, somnolence, increased sweating and decreased appetite. Nausea, the most common side effect, is usually mild and resolves within one week. After the first week of duloxetine treatment, the percentage of patients reporting new cases of nausea is similar to patients who receive placebo. Unlike venlafaxine, duloxetine is not associated with dose dependent hypertension. Duloxetine has no clinically relevant effect on the QT interval. In short-term trials (8-9 week trials) there is a small but significant amount of weight loss with Duloxetine. In a 6 month trial the mean weight gain for Duloxetine $60 \mathrm{mg} /$ day is no different from placebo.

Recent years has seen increasing interest in discontinuation symptoms i.e. symptoms that result from stopping medication. To investigate this phenomenon several trials had a design that included duloxetine being stopped abruptly, without taper, after which patients were followed up for 2 weeks. Discontinuation symptoms after stopping duloxetine were mild and transient with the most common symptom being dizziness.

In summary duloxetine appears well tolerated. Its side effects appear similar to those seen with SSRIs and venlafaxine. Preliminary data suggests that in some areas duloxetine may have a more favorable side effect profile than these comparators.

Published: 28 February 2006

doi:10.1186/1744-859X-5-S1-S22

Cite this article as: Haddad: Safety and tolerability of duloxetine adverse events and how to handle them. Annals of General Psychiatry 2006 5(Suppl 1):S22.

Consultant Psychiatrist, Manchester, UK 